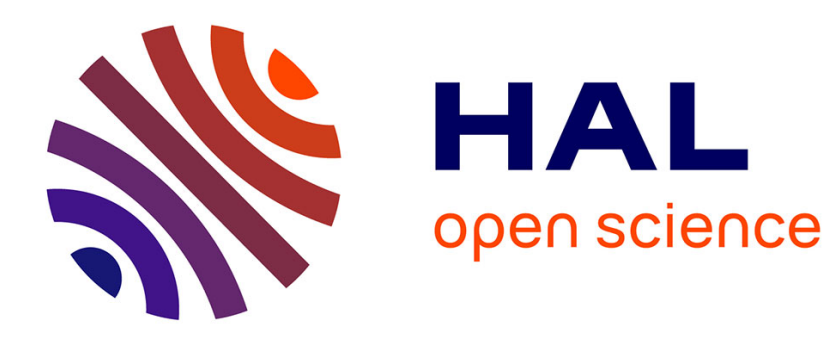

\title{
Étude ultrasonore des phénomènes critiques dans les gels
}

\author{
J.-C. Bacri, R. Rajaonarison
}

\section{- To cite this version:}

J.-C. Bacri, R. Rajaonarison. Étude ultrasonore des phénomènes critiques dans les gels. Journal de Physique Lettres, 1979, 40 (1), pp.5-8. 10.1051/jphyslet:019790040010500 . jpa-00231556

\section{HAL Id: jpa-00231556 https://hal.science/jpa-00231556}

Submitted on 1 Jan 1979

HAL is a multi-disciplinary open access archive for the deposit and dissemination of scientific research documents, whether they are published or not. The documents may come from teaching and research institutions in France or abroad, or from public or private research centers.
L'archive ouverte pluridisciplinaire $\mathbf{H A L}$, est destinée au dépôt et à la diffusion de documents scientifiques de niveau recherche, publiés ou non, émanant des établissements d'enseignement et de recherche français ou étrangers, des laboratoires publics ou privés. 


\title{
Étude ultrasonore des phénomènes critiques dans les gels
}

\author{
J.-C. Bacri et R. Rajaonarison \\ Laboratoire d'Ultrasons $\left({ }^{*}\right)$, Université Pierre-et-Marie-Curie, \\ Tour 13, 4 place Jussieu, 75230 Paris Cedex 05, France
}

(Reçu le 6 septembre 1978, révisé le 6 novembre 1978, accepté le 13 novembre 1978)

\begin{abstract}
Résumé. - Nous avons mesuré les variations d'atténuation et de vitesse ultrasonores dans un gel acrylique. En comparant ces résultats avec ceux obtenus dans le solvant pur $\left(\mathrm{H}_{2} \mathrm{O}\right)$, nous avons pu en déduire la constante élastique propre du gel et le coefficient de friction entre le réseau et le solvant. Une étude en fonction de la température nous a permis d'en déterminer leurs comportements critiques.
\end{abstract}

Abstract. - We have measured the ultrasonic velocity and attenuation in an acrylic gel. We have deduced the elastic constant and the frictional coefficient from the results obtained from the gel and those obtained from the pure solvent $\left(\mathrm{H}_{2} \mathrm{O}\right)$. We have observed a critical behaviour of these parameters.

Un gel est constitué d'un réseau de polymère réticulé en immersion dans un fluide. Récemment quelques expériences de diffusion de lumière [1], [2], [3], [4], ont été entreprises pour mettre en évidence les fluctuations de concentration du réseau de polymère. Tanaka et al. [5] ont observé une divergence critique de l'intensité de la lumière diffusée dans un gel polyacrylamide. L'intensité et le temps de corrélation augmentaient quand la température décroissait; et une divergence apparaissait à une certaine température $T_{\mathrm{s}}$.

Ces résultats s'expliquent en considérant les phénomènes critiques liés à la séparation de phases d'un mélange binaire, composé dans le cas d'un gel du fluide et du réseau polymérique; c'est-à-dire, un rétrécissement du réseau dans le fluide ambiant quand on s'approche de la température de transition. Le but de cette lettre est de présenter les mesures liées à la propagation d'une onde ultrasonore longitudinale traversant un gel en fonction de la température afin d'en déduire les paramètres propres au gel (constante élastique du réseau, coefficient de friction entre le réseau et le fluide ambiant) et d'étudier ces phénomènes aux voisinages de $T_{\mathrm{s}}$ (température spinodale) [6].

1. Théorie. - Pour simplifier le calcul, nous n'allons considérer que la propagation d'une onde longitudinale dans un gel. On appelle $r$ le déplacement

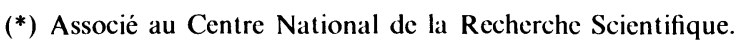

du réseau, $s$ celui du fluide, $\rho_{r}, E_{\mathrm{r}}$ et $\rho_{s}, E_{s}$ leur densité et leur module longitudinal respectifs. On obtient le système d'équations couplées suivant [1], [7], [8] :

$$
\left.\begin{array}{l}
\rho_{s} \ddot{s}=\left(\frac{4}{3} \eta+\xi\right) \nabla^{2} \dot{s}+E_{s} \nabla^{2} s+f(\dot{r}-\dot{s}) \\
c \rho_{r} \ddot{r}=E_{r} \nabla^{2} r+f(\dot{s}-\dot{r})
\end{array}\right\}
$$

$c$ est la concentration du polymère, $f$ le coefficient de friction entre le réseau et le fluide, $\eta$ et $\xi$ les coefficients de viscosité du fluide respectivement de cisaillement et de volume.

La résolution du système d'équations (1) aboutit à l'équation de dispersion suivante :

$$
\begin{aligned}
\left(\rho_{s}+c \rho_{r}\right) \omega^{2}=k^{2}\left(E_{s}+E_{r}\right)+ \\
+i \omega k^{2}\left(\frac{4}{3} \eta+\xi+\frac{c^{2} \rho_{r}^{2} E_{s}}{\rho_{s} f}\right)
\end{aligned}
$$

en considérant $E_{r} \ll E_{s}$ et $\omega \ll f / c \rho^{2}$.

Dans le cas où la partie imaginaire du vecteur d'onde est petite devant sa partie réelle, on obtient pour la vitesse dans le gel :

$$
v=\sqrt{\frac{E_{s}+E_{r}}{\rho_{s}+c \rho_{r}}} \text { et } \frac{\Delta v}{v} \simeq \frac{E_{\mathrm{r}}}{2 E_{s}} \text {. }
$$

$\Delta v$ représente la variation de la vitesse dans le gel. Quant à l'atténuation, elle comporte deux termes dus : l'un à la viscosité du fluide, l'autre à l'interaction entre le réseau et le solvant.

$$
\frac{\alpha}{v^{2}}=\frac{2 \pi^{2}}{\rho v^{3}}\left(\frac{4}{3} \eta+\xi+\frac{c^{2} \rho_{r} E_{s}}{\rho_{s} f}\right) \text {. }
$$


2. Méthode expérimentale. - Nous avons utilisé un système à impulsions ultrasonores classique. Les transducteurs ultrasonores employés sont des barreaux de quartz de coupe $\mathrm{X}$ en forme de clou. Le gel non encore réticulé est placé entre les deux quartz séparés par des cales de $2 \mathrm{~mm}$ à $100 \mu$ qui en définissent l'épaisseur (les fréquences variant de $100 \mathrm{MHz}$ à $200 \mathrm{MHz}$ ). La mesure de l'intensité du faisceau ultrasonore transmis en fonction de la température donne la variation de l'atténuation, tandis que celle de la phase permet d'accéder à la variation de la vitesse de phase. Ces quartz sont placés dans un four dont la température est régulée à $\pm 0,02^{\circ} \mathrm{C}$; celui-ci est mis dans un dewar dont la température peut varier de l'ambiante à $-100^{\circ} \mathrm{C}$. Une méthode impédométrique en ondes longitudinales [9] nous a permis de déterminer les vitesses du son dans l'eau et dans du gel en valeur absolue.

Nous utilisons un gel acrylique immergé dans l'eau. Pour l'obtenir d'une manière reproductible, nous commençons par préparer une solution mère $(30 \mathrm{~g}$ d'acrylamide, $0,8 \mathrm{~g}$ bis-acrylamide pour $100 \mathrm{ml}$ d' $\mathrm{H}_{2} \mathrm{O}$ ). Pour $10 \mathrm{ml}$ de gel, nous ajoutons successivement $3,33 \mathrm{ml}$ de solution mère, $0,330 \mathrm{ml}$ d'une solution de sulfate d'ammonium $(1 \mathrm{~g} / 100 \mathrm{ml})$ et $5 \mu \mathrm{l}$ de tétraméthyl-éthylène-diamine (TEMED). Le gel commence à réticuler quelques minutes après le mélange. Les expériences sont faites 24 heures plus tard.

3. Résultats expérimentaux. - Nous nous sommes intéressés au comportement critique du gel au voisinage de sa température spinodale $T_{\mathrm{s}}[6]$. Nous avons vu précédemment que les mesures ultrasonores permettent de déterminer les paramètres caractéristiques du gel $\left(E_{r}, f\right)$.

Nous avons mesuré la variation de la vitesse de phase dans le gel $\Delta v / v$ à $194 \mathrm{MHz}$ et $560 \mathrm{MHz}$. On observe sur la figure 1 que $\Delta v / v$ décroît quand la température décroît, mais un net changement de pente apparaît aux environs de $10^{\circ} \mathrm{C}$. La mesure impédométrique nous donne une valeur de la vitesse dans le gel de $1450 \pm 50 \mathrm{~m} / \mathrm{s}$ à $20^{\circ} \mathrm{C}$.

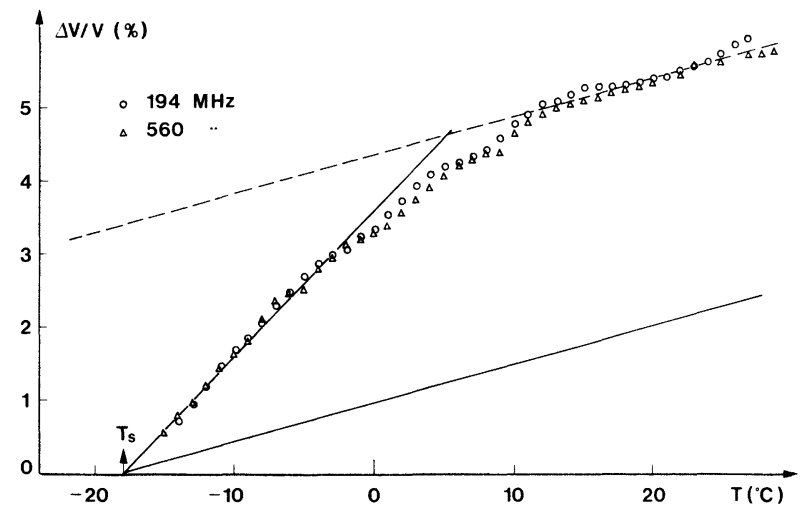

Fig. 1. - Variation de vitesse dans le gel en fonction de la température.

[Temperature dependence of velocity in the gel.]
Sur la figure 2 nous avons porté l'atténuation du gel moins celle de l'eau en fonction de la température (à $162 \mathrm{MHz}$ ). On voit clairement que $\alpha_{\text {gel }}-\alpha_{\text {eau }}$ diverge quand on s'approche de la transition.

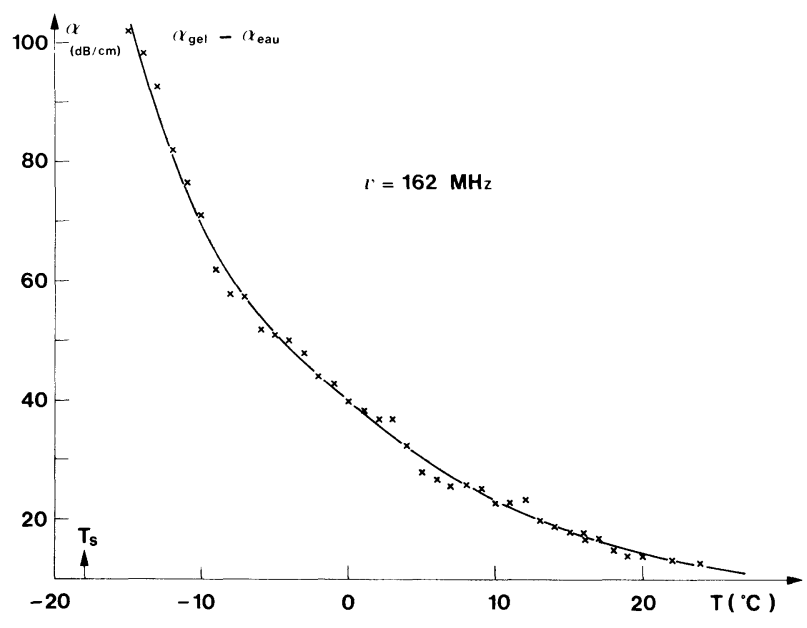

Fig. 2. - Différence entre l'atténuation dans le gel et celle dans l'eau en fonction de la température.

[Temperature dependence of the difference between attenuation in the gel and water.]

En fait il y a une incertitude sur la valeur de $T_{\mathrm{s}}$. Dans l'eau pure, la température de solidification dépend du volume de l'eau [10]. Pour un volume ayant la forme d'un cylindre d'une section de $3 \mathrm{~mm}$ et d'épaisseur de $1 \mathrm{~mm}$, nous pouvons retarder la solidification jusqu'à $-17^{\circ} \mathrm{C}$ et même jusqu'à $-26^{\circ} \mathrm{C}$ pour une épaisseur de quelques microns [11].

Pour des volumes équivalents, la température de solidification du gel varie de $-15^{\circ} \mathrm{C}$ à $-18^{\circ} \mathrm{C}$. Nous avons donc pris $T_{\mathrm{s}}=-18^{\circ} \mathrm{C}$.

4. Interprétation des résultats. - Tanaka et al. [5] ont calculé le module élastique longitudinal à partir de la pression osmotique en tenant compte de l'énergie élastique et de celle de mélange en utilisant les résultats de Flory. Ils ont trouvé que

$E_{r}=a\left(T-T_{\mathrm{s}}\right)$.

D’autre part, Kawasaki [12] a calculé la fonction de corrélation des fluctuations de concentration dans les mélanges binaires par une méthode de couplage de modes. Tanaka [6] a récemment appliqué ce calcul à celui de la séparation de phase dans un gel en considérant les fluctuations de concentrations du réseau polymérique. La valeur de la constante de diffusion est donnée par l'expression suivante

$D=\frac{k_{\mathrm{B}} T}{6 \pi \eta \xi}$

avec $\xi$ longueur de corrélation des fluctuations de concentration, $\eta$ viscosité de cisaillement du fluide, et celle de l'intensité de la lumière diffusée :

$I_{0} \sim \xi^{2}$. 
D'après les résultats des expériences en diffusion de lumière [1], [2], [3] et [4], la constante de diffusion s'écrit $D \sim E_{r} / f$ et $I_{0} \sim 1 / E_{r}$. On en déduit donc que $E_{\mathrm{r}} \sim \xi^{-2}$ et $f \sim \xi^{-1}$.

Dans le cadre d'une théorie de champ moyen,

$\xi=\xi_{0}\left(\frac{T-T_{\mathrm{s}}}{T}\right)^{-1 / 2}$

ce qui donne

$E_{\mathrm{r}} \sim T-T_{\mathrm{s}}$ et $f \sim\left(T-T_{\mathrm{s}}\right)^{-1 / 2}$.

Donc les variations de vitesse et d'atténuation en fonction de la température sont reliées de la manière suivante en utilisant les expressions (2) et (3) :

$\frac{\Delta v}{v} \sim T-T_{\mathrm{s}}$

$\Delta \alpha=\Delta \alpha_{\text {gel }}-\Delta \alpha_{\text {eau }} \sim\left(T-T_{\mathrm{s}}\right)^{-1 / 2}$.

Pour extraire la partie critique, i.e. $\Delta v(T) / v$ due à $E_{r}$, nous avons pris comme fond continu une droite dont la pente est fixée par la courbe expérimentale de $\Delta v(T) / v$ pour $T>10^{\circ} \mathrm{C}$. Les courbes de la figure 1 montrent clairement que la partie critique de $\Delta v(T) / v$ tend vers zéro linéairement quand $T \rightarrow T_{\mathrm{s}}$; donc $E_{r} \sim T-T_{\mathrm{s}}$ au voisinage de $T_{\mathrm{s}}$.

Pour vérifier la loi sur l'atténuation nous avons porté $\Delta \alpha$ en fonction de $T-T_{\mathrm{s}}$ en échelle logarithmique (Fig. 3). Une pente de $-0,5$ rend bien compte du phénomène critique.

$\mathrm{Du}$ point de vue de l'ordre de grandeur, la partie critique de $\left(\frac{\Delta v}{v}\right)_{T=10^{\circ} \mathrm{C}}-\left(\frac{\Delta v}{v}\right)_{T_{\mathrm{s}}}$ nous donne la valeur de $E_{\mathrm{r}} \sim 10^{9}$ dyne. $\mathrm{cm}^{-2}$; celle-ci apparaît très élevée par rapport aux résultats statiques $\left(10^{5}\right.$ dyne. $\left.\mathrm{cm}^{-2}\right)$ et ceux obtenus par diffusion de lumière $\left(5 \times 10^{5}\right.$ dyne. $\left.\mathrm{cm}^{-2}\right)$.

Quant à la valeur absolue de $\alpha_{\text {gel }}-\alpha_{\text {eau }}$ à la température ambiante, elle nous donne un ordre de grandeur de $f\left(f \sim 10^{10}\right.$ dyne. $\left.\mathrm{s} / \mathrm{cm}^{4}\right)$; cette valeur de $f$ est plus faible que celle trouvée en diffusion de lumière $\left(10^{11}\right.$ dyne.s $\left./ \mathrm{cm}^{4}\right)[4]$.

Une explication peut être donnée par l'apparition d'un temps de relaxation structurel : $E(\omega)$ et $f(\omega)$ s'écrivent :

$E(\omega)=\frac{E_{0}+(\omega \tau)^{2} E_{\infty}}{1+(\omega \tau)^{2}}$

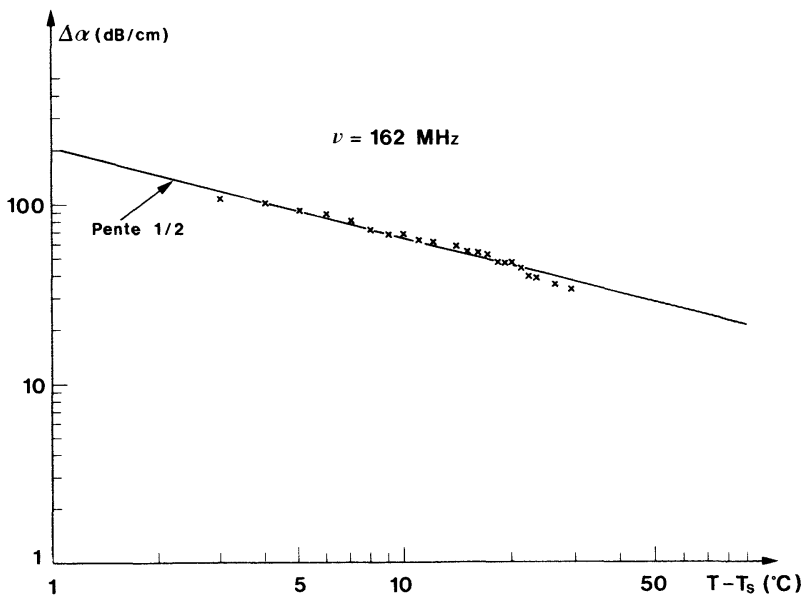

Fig. 3. - Différence entre l'atténuation dans le gel et celle dans l'eau en fonction de $T-T_{\mathrm{s}}$ en échelle log-log.

[Temperature dependence of the difference between attenuation in the gel and water on a $\log$-log scale.]

$f(\omega)=f_{0} \times \frac{1}{1+(\omega \tau)^{2}}$

et en prenant

$f_{0}=10^{11}$ dyne. $\mathrm{s} / \mathrm{cm}^{4}$,

$f(v=200 \mathrm{MHz})=10^{10}$ dyne. $\mathrm{s} / \mathrm{cm}^{4}$,

$E_{0}=10^{5}$ dyne. $\mathrm{cm}^{-2}$,

$E(v=1 \mathrm{MHz})=5 \times 10^{5}$ dyne. $\mathrm{cm}^{-2}$,

$E_{\infty}(v=200 \mathrm{MHz})=10^{9}$ dyne. $\mathrm{cm}^{-2}$,

les résultats sont cohérents avec $\tau \sim 5 \times 10^{-9} \mathrm{~s}$. Ce temps de relaxation est un peu plus grand que ceux observés dans les cristaux plastiques et les polymères fondus [14], mais beaucoup plus petit que ceux observés généralement dans les liquides. Les temps de relaxation trouvés dans les solutions diluées de polymères sont de l'ordre de quelque $10^{-9} \mathrm{~s}[15]$.

5. Conclusion. - Ces expériences montrent que la méthode ultrasonore à $200 \mathrm{MHz}$ permet de mesurer les paramètres caractéristiques des gels (constante élastique, coefficient de friction); elle permet également d'étudier leurs comportements critiques au voisinage de la température de séparation de phases. Des expériences en ondes transversales permettraient de bien compléter ces premiers résultats.

\section{Bibliographie}

[1] Tanaka, T., Hocker, L. O. and Benedek, G. B., J. Chem. Phys. 59 (1973) 5151.

[2] Munch, J. P., Candau, S., Duplessix, R., Picot, C. and Benort, H., J. Physique Lett. 35 (1974) L-239.

[3] Wun, K. L. and Carlson, F. D., Macromolécules 8 (1975) 191.
[4] Hecht, A. M. and Geissler, E., J. Physique 39 (1978) 631.

[5] Tanaka, T., Ishiwata, S. and Ishimoto, C., Phys. Rev. Lett. 38 (1977) 771.

[6] Tanaka, T., Phys. Rev. Lett. 40 (1978) 820.

[7] DE Gennes, P. G., Macromolécules 9 (1976) 587.

[8] DE Gennes, P. G., Macromolécules 9 (1976) 594. 
[9] BACRI, J. C., Thèse, Université Pierre-et-Marie-Curie, Paris, 1976.

[10] Rasmussen, D. H. and MacKensie, A. P., J. Chem. Phys. 59 (1973) 5003.

[11] BaCri, J. C. et Rajaonarison, R., à paraître.

[12] Kawasaki, K., Ann. Phys. 61 (1970) 1.
[13] Tanaka, T., Phys. Rev. $A 17$ (1978) 763.

[14] Huang, Y. Y., Friedman, E. A., Andrews, R. D. and Hart, T. R., Proc. of the 2nd Internat. Conf. on Light Scattering in Solids, Paris July 1971, p. 488.

[15] Pugh, D. and Jones, D. A., Polymer 19 (1978) 1008. 